\title{
Satisfação e situação profissional: um estudo com professores nos primeiros anos de carreira
}

Mariana Gaio Alves'

Nair Rios Azevedo'

Teresa N. R. Gonçalves'

I- Universidade Nova de Lisboa,

\section{Resumo}

O ingresso em uma profıssão e as motivações para a sua escolha, os primeiros anos de carreira e o estatuto da profıssão, em especial a docente, têm sido objeto de diversas investigações. Porém, tanto no caso português como no âmbito internacional, a inserção profissional dos professores tem sido negligenciada pelas pesquisas acadêmicas. Assim sendo, neste artigo, propomo-nos a contribuir com o conhecimento do processo de inserção profissional dos docentes de ensino básico e secundário, por meio da análise do grau de satisfação na profissão, dos aspectos mais e menos valorizados no trabalho, bem como da situação profissional em dois períodos, após um ano e depois de cinco anos de conclusão da licenciatura. Consideramos os licenciados em educação em comparação com todos os licenciados das universidades de Lisboa e Nova de Lisboa. Os dados apresentados permitem constatar que, nas dimensões estudadas, não existem diferenças significativas entre os licenciados com relação aos aspectos analisados. Das particularidades do grupo de licenciados em educação que participaram da pesquisa, destacamos: a ligeira saliência que assumem os aspectos altruístas; uma satisfação com a profıssão ligeiramente maior; uma tendência à precarização dos vínculos contratuais e uma disposição para auferir em média rendimentos ligeiramente inferiores. Como considerações finais deste artigo, destacamos a necessidade de aprofundamento no estudo do modo como as condições de exercício da profissão influenciam o desenvolvimento dos professores em fase de inserção profissional. Além disso, salientamos a pertinência de que melhores formas de apoiar a aprendizagem profissional sejam exploradas, bem como a construção da identidade profissional dos professores em início de carreira.

\section{Palavras-chave}

Satisfação profissional - Inserção profissional - Valores no trabalho - Profissão docente.

Lisboa, Portugal.

Contatos: mga@fct.unl.pt,

n.azevedo@fct.unl.pt,

tprg@fct.unl.pt 


\title{
Satisfaction and professional status: a study of teachers in the early years of their careers
}

Mariana Gaio Alves'

Nair Rios Azevedo'

Teresa N. R. Gonçalves'

\begin{abstract}
The entry into a profession and the motivations for choosing it, the early years of one's career and statute of the profession, in particular the teaching one, have been the subject of several investigations. However, both in Portugal and abroad, the professional insertion of teachers has been neglected by academic research. Therefore, in this article, we propose to contribute to the knowledge of the professional insertion of primary and secondary teachers by examining the degree of satisfaction in the profession, the most and least valued aspects at work, as well as the professional status in two periods, one year and five years after completing the degree. We consider graduates in education compared to all graduates of the universities of Lisbon and New Lisbon. The data presented allows us to state that, in the dimensions studied, there are no significant differences between graduates regarding the aspects analyzed. The particularities of the group of graduates in education who participated in the research include: a slight prominence of altruistic aspects; a slightly greater satisfaction with the profession; a trend towards casualization of employment contracts and a willingness to earn on average slightly lower income. In conclusion, we highlight the need for further study of how professional conditions influence the development of teachers in the phase of professional insertion. Furthermore, we stress that better ways to support professional learning should be explored, as well as the construction of teachers' professional identity in early career.
\end{abstract}

\section{Keywords}

Professional satisfaction - Professional insertion - Values at work - Teaching profession.

I- Universidade Nova de Lisboa,

Lisboa, Portugal.

Contacts: mga@fct.unl.pt,

n.azevedo@fct.unl.pt,

tprg@fct.unl.pt 


\section{Introdução}

A entrada na profissão e as motivações para a sua escolha, os primeiros anos de carreira e o estatuto da profissão docente têm sido objeto de diversas investigações. Desde os anos 60 que se tem desenvolvido um corpo significativo de pesquisa sobre as motivações, crenças e desenvolvimento inicial de carreira no sentido de tentar entender as razões que estão na origem da escolha da profissão docente, as representações/percepções da profissão por parte dos professores principiantes ou dos candidatos a professores e quais as suas aspirações relativamente ao desenvolvimento da carreira (BROOKHART; FREEMAN, 1992; MALDEREZ et al., 2007; RICHARDSON; WATT, 2005; RINKE, 2008; WANG, 2004; WATT; RICHARDSON, 2008).

Neste artigo, propomo-nos a contribuir para o conhecimento do processo de inserção profissional dos professores. A investigação a esse respeito, no âmbito do ensino superior, começou a ser desenvolvida em Portugal na década de 1990. Em outros países, como Canadá, França e Reino Unido, os estudos datam desde os anos 1970 (ALVES, 2010).

Porém, no caso português, desconhecemos pesquisas sobre a inserção profissional que analisem especificamente os diplomados em licenciaturas em educação, permitindo a caracterização dos seus primeiros anos de carreira. Essa escassez de estudos não é uma característica peculiar do contexto a que nos referimos, uma vez que, no plano internacional, outros autores indicam essa tendência. Como colocam Akkari e Tardif (2011, p. 125), mesmo a carreira docente sendo um domínio bastante explorado:

[...] a inserção profissional, como fase de procura de um emprego no ensino, tem sido por muito tempo um fenómeno negligenciado pela pesquisa em educação.

Nossa colaboração com as pesquisas da área é estudar a satisfação no trabalho, procurando explorar tanto a dimensão mais objetiva da inserção profissional (enquanto trajetória de acesso ao emprego), como a dimensão mais subjetiva, relativa ao modo como essa etapa é vivida pelos indivíduos. Para tal, os objetivos do estudo consistem em analisar o grau de satisfação na profissão, os aspectos mais e menos valorizados no trabalho, bem como a situação profissional um ano e cinco anos após a conclusão da licenciatura, considerando os licenciados em educação em comparação com outros licenciados.

Mobilizamos dados empíricos dos diplomados das universidades de Lisboa e Nova de Lisboa, comparando a situação dos licenciados em educação com a dos licenciados de todas as áreas de formação. Os dados apresentados referem-se a licenciados em educação, na grande maioria professores em início de carreira (primeiros cinco anos após a conclusão da formação inicial), abrangendo toda a primeira fase do ciclo de vida dos professores e o início da segunda fase, tal como são descritas por Huberman (1989).

De acordo com o autor, a primeira fase corresponde à entrada na profissão e aos primeiros três anos de carreira, caracterizandose pela exploração do exercício profissional e entusiasmo dos principiantes. A segunda fase, entre o quarto e o sexto ano de exercício profissional, distingue-se por um sentimento de maior conforto no trabalho e pela estabilização e consolidação profissionais.

Nossa abordagem da satisfação profissional e do que é mais valorizado no trabalho dos professores assenta-se no pressuposto de que as condições de exercício da profissão nos primeiros anos influenciam significativamente o processo de aprendizagem profissional (AKKARI; TARDIF, 2011) e o processo de construção da identidade profissional (FLORES; DAY, 2006). A exploração dessas temáticas é particularmente pertinente, tendo em conta o fato de que a satisfação pessoal e a realização de si são desafios importantes para os sujeitos no desenrolar da suas trajetórias de trabalho e emprego (AKKARI; TARDIF, 2011). 
Com efeito, concordamos com a ideia de que a inserção profissional deve ser entendida como um conceito multidimensional (AKKARI; TARDIF, 2011; ALVES, 2005), o que significa privilegiar não apenas a questão do acesso ao emprego por parte dos diplomados (tradicionalmente abordada nesse domínio de pesquisa), mas também os aspectos referentes à inserção profissional enquanto período de aprendizagem profissional e construção de identidade.

Tendo em vista os objetivos da investigação, a nossa análise será enquadrada por uma reflexão acerca da motivação e o estatuto profissional dos professores e sobre a satisfação profissional. Pretendemos, a partir da interpretação dos dados, identificar pistas para futuras investigações a respeito da inserção profissional dos licenciados em educação e condições de exercício da profissão.

\section{Motivação e estatuto profissional dos professores}

A temática da motivação tem sido entendida como se referindo tanto aos motivos que levam à escolha da profissão, como aos que se relacionam com a permanência na mesma ou a desistência e opção por outra carreira/ profissão (JOHNSON; BIRKELAND, 2003).

Relativamente à motivação de entrada na profissão, estudos desenvolvidos em vários contextos de formação de professores indicam que as razões mais apontadas para a escolha da profissão estão relacionadas com o exercício da criatividade, o gosto em trabalhar com os mais novos e o desejo de desenvolver uma atividade profissional socialmente útil (HAYES, 1990; STIEGELBAUER, 1992).

Mais recentemente, um estudo desenvolvido no Reino Unido (HARGREAVES et al., 2006) compara as razões apontadas por professores em exercício e por professores estagiários para a escolha da profissão. Os aspectos referidos por ambos os grupos foram: realização pessoal, um trabalho com um estatuto confortável, desenvolvimento pessoal e profissional, dar às crianças a oportunidade de um bom começo e ser um trabalho desafiante.

De um modo geral, os investigadores têm agrupado as razões dos professores para a escolha da profissão em razões extrínsecas e intrínsecas à profissão e, ainda, razões altruístas (SUMMERHILL; MYRNA, 1998; YONG, 1995). Reconhece-se, no entanto, que as razões para se tornar professor podem variar e que a estrutura das motivações para essa escolha é multidimensional (BROOKHART; FREEMAN, 1992; REEVE; BOLT; CAI, 1999; SPITTLE; JACKSON; CASEY, 2009).

A motivação dos professores para escolher a profissão e optar pela profissionalização pode também ser vista em função dos contextos políticos e administrativos de cada país. No caso português, com um sistema educativo bastante centralizado, o Ministério da Educação (ME) exerce uma forte influência sobre os professores e o ensino, assim como sobre as condições que definem (encorajando ou desencorajando) a profissionalização. Como afirmam Hildebrandt e Eom (2011), a natureza da certificação profissional dos professores em sistemas educativos centralizados pode tornar a motivação para a profissionalização discutível ou controversa, permitindo aos professores pouca escolha, uma vez que as trajetórias de carreira são rígidas. Também as áreas disciplinares de docência são um fator importante a ter em conta, como demonstra a falta de professores em algumas áreas (como a matemática ou ciência) em alguns países da Europa (EREN; TEZEL, 2010; ZUMWALT; CRAIG, 2008).

A motivação de escolha da profissão pode ainda ser relacionada com as questões do estatuto profissional dos professores face a outras profissões. Na verdade, o processo de profissionalização define um conjunto de traços comuns e de atributos, valores, experiências, requisitos de qualificação, conhecimentos, competências e práticas partilhados que, simultaneamente, atribuem coesão interna a um grupo profissional e permitem a sua diferenciação 
e comparação com outros grupos, contribuindo para a construção da identidade profissional.

A distinção entre profissões tradicionais, como aquelas que correspondem a um conjunto de requisitos normativos baseados na qualificação, organização ou competência, e as semiprofissões ou profissões incompletas, como aquelas que carecem de alguns dos marcadores necessários à definição de uma profissão entendida no sentido tradicional (JÜTTE; NICOLL; OLESEN, 2011), é de particular importância no caso dos professores.

No estudo de Hargreaves et al. (2006) foi pedido aos professores e outros grupos associados (pais, decisores locais, media, entre outros) que relacionassem afirmações acerca de profissões com um estatuto elevado e sua aplicação à profissão docente. Os resultados do estudo demonstram que a profissão docente é vista como mais semelhante a uma profissão de elevado estatuto no que diz respeito a "ser uma profissão responsável de alto desempenho”, mas que continuam a existir diferenças significativas entre a profissão docente e as profissões consideradas de elevado estatuto no que se refere a alguns fatores definidos como recompensa-respeito e controle-regulação.

0 estatuto profissional dos professores face a outras profissões assume relevância, uma vez que, quando comparada com as profissões tradicionais (advogados, médicos, arquitetos), a carreira docente é muitas vezes entendida como uma quase-profissão (DARLING-HAMMOND, 1999), uma service profession (MEYERS, 2008), ou um ofício (PRATTE; RURY, 1991).

Mesmo com todos os progressos feitos no processo de profissionalização dos professores, a definição da profissão docente como uma profissão de pleno direito, principalmente quando comparada com as profissões tradicionais, tem sido um desafio em todo o mundo, apesar das diferentes realidades (FULLAN, 2001; HARGREAVES, 2001; DAY; SACHS, 2004).

0 estatuto profissional e social da profissão docente pode representar um fator relevante em termos de motivação e satisfação profissional. Assim sendo, nosso estudo pretende dar um contributo para a compreensão das semelhanças e diferenças entre a perspectiva dos docentes e a de outros profissionais.

\section{Satisfação profissional}

Para além das motivações de escolha e do estatuto profissional da atividade docente, a permanência na profissão tem também sido relacionada com a satisfação no trabalho. Ela é tratada enquanto constructo e, como tal, tem sido entendida de diferentes maneiras e abordada segundo pontos de vista conceptualmente diferenciados. Alguns autores têm mesmo sugerido um vazio teórico na análise da satisfação profissional, o que pode explicar interpretações contraditórias sobre o que significa estar satisfeito com o trabalho e os fatores que para isso contribuem (WEISS, 2002).

Em sentido mais abrangente, a satisfação profissional vem sendo considerada como a apreciação positiva ou negativa que é feita sobre o trabalho que se exerce (WEISS, 2002; LOCKE, 1969). Nessa avaliação, são incorporados elementos de natureza cognitiva e afetiva, permitindo que a pessoa emita um julgamento onde estão integradas razões mais ou menos objetivas. Por outro lado, a comparação entre o que se consegue obter na atividade profissional com aquilo que dela era esperado é apontado também como um fator de influência na satisfação com o trabalho (LOCKE, 1969), conjugando a apreciação da situação real de trabalho com as expectativas anteriormente construídas.

Um dos modelos mais utilizados na análise da satisfação com o trabalho é o proposto por Herzberg (1996), segundo o qual são integrados dois conjuntos de fatores explicativos: por um lado, os fatores motivacionais (reconhecimento, realização e conquistas; a atividade desenvolvida; a responsabilidade; e a progressão na carreira) que, quando presentes, aumentam o sentimento de satisfação, mantêm a motivação e tendem 
a contribuir para um melhor desempenho; por outro lado, os fatores de higiene (hygiene factor) ou de contexto (políticas institucionais, de gestão e organização; condições de trabalho, suporte e supervisão; relações interpessoais; remuneração; status; segurança e estabilidade) que, na sua ausência, contribuem para a nãosatisfação. Uns e outros são responsáveis pela satisfação ou insatisfação, na medida em que a presença de motivadores (fatores intrínsecos) contribui para a satisfação, enquanto a ausência de fatores de contexto (extrínsecos) contribui para a não satisfação (FURNHAM, 1992; FURNHAM; ERACLEOUS; PREMUZIC, 2009).

De um modo geral, tem sido adoptada uma dupla perspectiva de avaliação da satisfação com o trabalho (SPECTOR, 1997; PEDRO, 2011): uma perspectiva mais global, em que a atividade profissional é considerada como um todo, refletindo-se no modo como o profissional se sente em relação ao trabalho de uma forma geral; e uma perspectiva multidimensional, relativa aos diversos fatores que podem ser considerados. Neste trabalho, o estudo da satisfação é abordado na perspectiva mais global.

Relativamente à satisfação dos professores com a profissão, os trabalhos têm seguido os quadros teóricos adoptados no estudo da satisfação com o trabalho e têm indicado que a satisfação é resultado de um conjunto de fatores inter-relacionados (SKAALVIK; SKAALVIK, 2011). No entanto, e tal como nos estudos da satisfação profissional em geral, não parece haver consenso sobre o modo de avaliar o constructo. Ele tem sido tratado relativamente a fatores específicos (o grau em que os professores estão satisfeitos com determinados aspectos do seu exercício profissional) e enquanto avaliação global (um sentido geral de satisfação/insatisfação) (MOE; PAZZAGLIA; RONCONI, 2010).

No que se refere à atividade docente, tem sido apontado um progressivo aumento de insatisfação, que é relacionado com uma menor motivação, envolvimento e empenho dos professores, menor percepção de controle, comprometimento e profissionalismo (CORDEIRO-
ALVES, 1991; HARKER et al., 1998; JESUS, 2005; PEDRO; PEIXOTO, 2006; PEDRO, 2011).

Parece consensual o reconhecimento do mal-estar manifestado pelos professores, relacionado com um aumento de exigências e pressões sobre eles exercidas e associadas com uma maior degradação nas condições de trabalho e exercício docente (JESUS, 2005; PEDRO, 2011). Se, por um lado, o trabalho do professor é fonte de realização e satisfação, por outro, está impregnado de motivos de stress e desgaste (JOHNSON et al., 2005).

A investigação tem demonstrado que a maior satisfação dos professores surge relacionada com a docência propriamente dita, enquanto que a menor satisfação parece decorrer das condições sociopolíticas de trabalho (PEDRO, 2011). Se a relação com os estudantes é apontada como uma das razões de maior satisfação, a falta de apoio, a baixa remuneração, o pouco reconhecimento social e o excessivo trabalho administrativo têm sido referidos como fontes de insatisfação (PERKING, 1991; CLARKE; KEATING, 1995).

Apesar da escassa investigação a respeito da realidade dos professores portugueses, estudos conduzidos em outros países exploram os fatores que aparecem relacionados com a satisfação dos professores. Por exemplo, é sugerida uma relação entre os anos de exercício profissional e a satisfação no trabalho, havendo indicação de que professores mais experientes manifestam maior índice de satisfação (LIU; RAMSEY, 2008).

Outros fatores tidos em conta referem-se à idade ou ciclos de vida (HUBERMAN, 1989), à mudança de atitudes para com a profissão ao longo da carreira e aos níveis de burnout (ZABEL; ZABEL, 2001). Embora não exista consenso sobre a influência dos fatores etários ou de experiência profissional (HILDBRANDT; EOM, 2011), muitos estudos identificam diferenças de motivação entre professores de distintas faixas etárias (KUHLEN; JOHNSON, 1952).

Outros trabalhos indicam uma maior satisfação das professoras quando comparadas com os seus pares masculinos (LIU; RAMSEY, 
2008; MENON; PAPANASTASIOU; ZEMBYLAS, 2008). Relativamente ao grau de ensino em que lecionam, são os professores do $1^{\circ}$ ciclo aqueles que manifestam maior satisfação, ao contrário dos professores do ensino secundário, que indicam uma menor satisfação com o trabalho (MENON; PAPANASTASIOU; ZEMBYLAS, 2008).

Rhodes, Nevill e Allan (2004) inquiriram um conjunto de professores do Reino Unido, procurando identificar os fatores relacionados com a maior satisfação ou insatisfação. 0 volume de trabalho, as tarefas administrativas, o pouco reconhecimento social e o comportamento dos alunos foram as razões mais apontadas para a insatisfação. As razões de maior satisfação, por sua vez, foram a boa relação com os colegas, o bom ambiente de sala de aula, o incentivo à inovação e realização, bem como o reconhecimento pela liderança. Em síntese, como Ruivo et al. (2008, p. 9) referem:

[...] o prestígio, areputação, oreconhecimento, a realização e o desenvolvimento pessoal e profissional, a necessidade de crescimento, são valores fundamentais tidos em conta pela maioria dos teóricos.

Em pesquisas da Organização para a Cooperação e Desenvolvimento Econômico - OCDE envolvendo diferentes países (OCDE, 2009; 2011) são referidos a avaliação do trabalho dos professores e o feedback que recebem como tendo uma influência positiva na satisfação que esses manifestam, mais concretamente no sentido de autoeficácia. Também o clima de escola, a cooperação entre os colegas e a relação com os estudantes aparecem relacionados com o sentimento de satisfação.

No que diz respeito à realidade portuguesa em comparação com outros países, alguns estudos (JESUS, 1998; PINTO et al. 2003; OCDE, 2011) têm revelado que os professores portugueses são os que manifestam uma menor satisfação com a profissão e maior stress profissional. Dados nacionais (RUIVO et al., 2008), com uma amostra de educadores de infância e professores do Ensino Básico e Secundário (EBS), indicam que uma grande maioria dos inquiridos revelaram uma preocupação com o futuro profissional e dizem não estarem satisfeitos com o (pouco) apoio pedagógico que lhes é dado pelo Ministério da Educação. A maioria manifesta o pouco reconhecimento social do seu trabalho, que se traduz em um vencimento que sentem não corresponder ao esforço despendido. Apesar de afirmarem o gosto pela profissão, dizem sentir-se "merecedores de mais e melhor reconhecimento, [e] melhor vencimento" (RUIVO et al., 2008, p. 27).

\section{Metodologia}

Os dados apresentados e discutidos neste artigo resultam de um questionário respondido em novembro de 2010 por uma amostra representativa de licenciados das Universidades de Lisboa e Nova de Lisboa que terminaram os respetivos cursos no ano letivo 2004/051. Procuramos destacar as características do grupo de licenciados em educação, contrastando-as com a amostra total constituída por graduados de todas as áreas disciplinares.

A amostra global é constituída por 1004 licenciados e é estatisticamente representativa do universo de 4290 licenciados das Universidades de Lisboa e Nova de Lisboa em 2004/05. Os inquiridos distribuem-se por diferentes domínios disciplinares: artes e humanidades $(21,5 \%)$; saúde $(12,5 \%)$; ciências físicas (12\%); educação (10\%); direito (9,8\%); ciências da vida (8,5\%); ciências sociais e jornalismo (7,7\%); economia e gestão $(6,7 \%)$; matemática, estatística e computadores (6,5\%); e engenharia, indústria transformadoras e construção $(4,8 \%)$. Quase todos os inquiridos são cidadãos portugueses $(99,8 \%)$, sendo que a maioria é mulher (64,3\%). A média de idades

1- 0 questionário foi realizado no âmbito de um projeto apoiado pela Fundação para a Ciência e Tecnologia de Portugal, entre 2010 e 2012, com 0 título "Percursos de inserção de licenciados: relações objetivas e subjetivas com o trabalho" (referência PTDC/CS-SOC/104744/2008). 
no momento da inquirição é de 31,94 anos, indiciando que a maioria frequentou o ensino superior com cerca de 20 anos.

Neste artigo, os resultados globais (1004 licenciados) são confrontados com os dados referentes aos licenciados em educação (104 indivíduos). Esse grupo mais reduzido inclui licenciados em ciências de educação (9) e licenciados em cursos de formação de professores de várias disciplinas: ensino de física e química (18), matemática (16), biologia e geologia (12) e ciências da natureza (5); e ainda formação educacional em línguas e literaturas modernas (35), línguas e literaturas clássicas (4), história (3) e filosofia (2).

Verifica-se que, no momento da inquirição, os respondentes licenciados em educação são na maioria professores EBS (82,9\%), distribuindo-se os restantes pela docência no ensino superior (5,7\%), pela situação de bolseiros (5,7\%), pela docência no $1^{0}$ ciclo do ensino básico $(2,9 \%)$ e no ensino especial, artístico ou desportivo (2,9\%).

A formação acadêmica e profissional dos professores EBS era assegurada pelas universidades numa lógica integrada ou sequencial (LEITE, 2005). No primeiro caso, a formação pedagógica e o estágio estavam integrados com a formação científica nos planos de estudos das licenciaturas, enquanto no segundo caso se completava primeiro a formação científica e, em seguida, a formação pedagógica e estágio.

Tendo em conta os nossos objetivos, mobilizamos os dados que permitem caracterizar a situação profissional dos licenciados em dois momentos da trajetória após a graduação: um ano e cinco anos depois da conclusão da licenciatura. Analisamos as respostas a perguntas incluídas no questionário que permitem saber, nos dois momentos considerados, qual a situação dos licenciados no mercado de trabalho, qual o seu tipo de vínculo contratual e nível de rendimento. Também foram mobilizados os dados referentes à satisfação com o percurso profissional e com a profissão, bem como a apreciação dos inquiridos sobre os aspectos mais e menos valorizados no trabalho. Solicitou-se aos respondentes que se pronunciassem, em uma escala de 1 a 10 , sobre a sua concordância ou sobre a importância atribuída a essas dimensões, correspondendo o 1 a menos satisfeito ou menos importante.

Os resultados empíricos que em seguida se apresentam referem-se a um conjunto de indivíduos que se licenciaram antes das mudanças curriculares e pedagógicas que vieram a ser introduzidas no ensino superior, na sequência do processo de Bolonha. Note-se, também, que as suas trajetórias após a conclusão da licenciatura se desenvolveram antes do agravamento da crise econômica que teve lugar em 2011. Nessas condições, traça-se um retrato da situação na primeira década do século XXI, que poderá ser objeto de profundas alterações. No entanto, constitui um ponto de partida para analisar modificações em curso e para refletir sobre a inserção profissional dos professores.

\section{Apresentação e discussão de dados}

\section{a) Satisfação profissional}

Com o objetivo de analisar o grau de satisfação profissional dos licenciados em educação e de outros licenciados, os dados do questionário permitem considerar quatro dimensões de análise: satisfação com o percurso profissional, satisfação com a atividade profissional no momento da inquirição (cinco anos após a licenciatura), adequação das funções profissionais à área de formação em que se licenciou e correspondência do trabalho atual face às expectativas quando se concluiu a licenciatura.

No que refere à satisfação com o percurso profissional, até ao momento da inquirição (quadro 1), constata-se que os licenciados se manifestaram muito positivamente (cerca de 7 numa escala de 1 a 10), não se registrando diferenças entre a apreciação dos licenciados 
em educação e a apreciação do conjunto dos licenciados inquiridos.

Quadro 1 - Grau de satisfação com o percurso profissional até ao momento da inquirição

\begin{tabular}{c|c|c}
\hline Satisfação & $\begin{array}{c}\text { Todos os } \\
\text { licenciados }\end{array}$ & $\begin{array}{c}\text { Licenciados em } \\
\text { Educação }\end{array}$ \\
\hline Valor médio & 7,2 & 7,3 \\
\hline Desvio-padrão & 1,9 & 1,8 \\
\hline
\end{tabular}

Fonte: dados da pesquisa

Quando questionados sobre a satisfação com a atividade profissional, os licenciados manifestaram-se também muito positivamente (quadro 2). Porém, há uma ligeira diferença entre os licenciados em educação e o conjunto de todos os licenciados, sendo que os primeiros afırmam em média um grau mais elevado de satisfação.

Quadro 2 - Grau de satisfação com a atividade profissional no momento da inquirição

\begin{tabular}{|c|c|c|}
\hline Satisfação & $\begin{array}{l}\text { Todos os } \\
\text { licenciados }\end{array}$ & $\begin{array}{l}\text { Licenciados em } \\
\text { Educação }\end{array}$ \\
\hline Valor médio & 7,8 & 8,2 \\
\hline Desvio-padrão & 1,8 & 1,6 \\
\hline
\end{tabular}

Fonte: dados da pesquisa

Relativamente à adequação ou correspondência entre a formação na licenciatura e a atividade profissional, constatou-se que os licenciados em educação manifestaram-se mais positivamente do que o conjunto dos licenciados. Como se observa nos quadros 3 e 4, a apreciação da adequação entre a área de licenciatura e as funções profissionais, bem como da correspondência entre as expectativas no momento da conclusão da licenciatura e o trabalho atual atingiram valores médios que tendem a ser um pouco mais elevados no caso dos licenciados em educação.
Quadro 3 - Grau de adequação das funções profissionais à área em que se licenciou

\begin{tabular}{|c|c|c|}
\hline Satisfação & $\begin{array}{c}\text { Todos os } \\
\text { licenciados }\end{array}$ & $\begin{array}{l}\text { Licenciados em } \\
\text { Educação }\end{array}$ \\
\hline Valor médio & 7,3 & 8,4 \\
\hline Desvio-padrão & 2,9 & 2,4 \\
\hline
\end{tabular}

Fonte: dados da pesquisa

Quadro 4 - Grau de correspondência do trabalho atual relativamente às expectativas que tinha quando concluiu a licenciatura

\begin{tabular}{c|c|c}
\hline Correspondência & $\begin{array}{c}\text { Todos os } \\
\text { licenciados }\end{array}$ & $\begin{array}{c}\text { Licenciados em } \\
\text { Educação }\end{array}$ \\
\hline Valor médio & 6,7 & 7,1 \\
\hline Desvio-padrão & 5,2 & $\vdots$ \\
\hline
\end{tabular}

Fonte: dados da pesquisa

Em síntese, registraram-se diferenças muitos ligeiras ${ }^{2}$ entre as avaliações dos licenciados em educação e as dos outros licenciados. Nos casos em que se registraram diferenças, verifica-se entre os licenciados em educação um grau médio de satisfação com a atividade profissional mais elevado, uma apreciação mais favorável da correspondência face a expectativas prévias, bem como uma apreciação mais favorável da adequação entre a formação inicial e a atividade profissional.

\section{b) Aspectos mais e menos valorizados no trabalho}

Procurando compreender quais os aspectos mais e menos valorizados no trabalho, os licenciados foram questionados relativamente à atividade profissional que desenvolviam no momento da inquirição e sobre o trabalho em geral. Tendo por base categorizações já existentes sobre os valores no trabalho (SUMMERHILL; MYRNA, 1998; YONG, 1995; CHAVES, 2010), consideramos as categorias de

\footnotetext{
2- Trata-se de diferenças muito ligeiras, sem significado estatístico.
} 
carreira, status, aspectos financeiros, realização pessoal e altruísmo.

Relativamente à atividade profissional no momento da inquirição, pode-se observar (quadro 5) que elementos relacionados com a carreira apresentam valores médios de importância menos elevados, incluindo-se aqui a segurança e estabilidade no trabalho e as oportunidades de progressão, não existindo diferença significativa entre os licenciados em educação e os restantes licenciados. Também o aspecto trabalho que permite condição social privilegiada (status) assume uma importância menos elevada, particularmente entre os licenciados em educação face ao conjunto de todos os licenciados.

$\mathrm{Na}$ categoria relativa aos aspectos financeiros, é notória a diferença na importância atribuída aos rendimentos elevados que o trabalho permite (pouco valorizada), face à autonomia financeira alcançada pelo exercício profissional (muito valorizada). Não existem variações entre licenciados em educação e outros licenciados. 0 fato de os inquiridos serem recém-licenciados pode explicar que valorizem a autonomia financeira alcançada nos primeiros anos de profissão, apesar de considerarem que os rendimentos auferidos não são muito elevados.

Os aspectos mais valorizados pelos inquiridos dizem respeito à realização pessoal, na qual se incluíram os itens trabalho interessante, trabalho que permite novos conhecimentos e trabalho que permite autonomia. Nesses aspectos não se observam diferenças na importância atribuída pelos licenciados em educação e por todos os licenciados.

Refira-se, ainda, que a categoria altruísmo, que inclui aspectos como trabalho que permite ajudar os outros e trabalho que é útil à sociedade, é muito valorizada. No caso dos licenciados em educação, são mesmo os aspectos a que atribuem maior importância, o que, em nosso entender, pode estar relacionado com o exercício de uma profissão que visa, fundamentalmente, a promover o desenvolvimento e aprendizagem das pessoas.

Quadro 5 - Aspectos que mais valorizam na atividade profissional que desenvolvem

\begin{tabular}{|c|c|c|c|}
\hline Categorias & $\begin{array}{l}\text { Aspectos valorizados } \\
\text { Trabalho... }\end{array}$ & $\begin{array}{l}\text { Todos os licenciados } \\
\text { Média (desvio-padrão) }\end{array}$ & $\begin{array}{l}\text { Licenciados em Educação } \\
\text { Média (desvio-padrão) }\end{array}$ \\
\hline \multirow{2}{*}{ Carreira } & seguro e estável & $6,6(2,8)$ & $5,3(2,8)$ \\
\hline & que permite oportunidades de progressão & $6(2,5)$ & $5,1(2,6)$ \\
\hline \multirow{2}{*}{ Financeira } & que permite rendimentos elevados & $5,8(2,3)$ & $5,2(2,3)$ \\
\hline & que permite autonomia financeira & $8,6(2,1)$ & $8,8(1,9)$ \\
\hline Status & que permite condição social privilegiada & $5,6(2,3)$ & $4,8(2,3)$ \\
\hline \multirow{3}{*}{$\begin{array}{c}\text { Realização e /ou } \\
\text { desenvolvimento pessoal }\end{array}$} & que é interessante & $7,8(1,9)$ & $8,3(1,6)$ \\
\hline & que permite novos conhecimentos & $8(1,9)$ & $8(1,8)$ \\
\hline & que permite autonomia & $7,8(1,9)$ & $7,8(1,9)$ \\
\hline \multirow{2}{*}{ Altruísmo } & que permite ajudar os outros & $7,7(2,2)$ & $8,5(1,6)$ \\
\hline & que é útil à sociedade & $8,1(2)$ & $9,1(1,5)$ \\
\hline
\end{tabular}

Fonte: dados da pesquisa 
Quando questionados sobre o mesmo conjunto de aspectos, mas se reportando ao trabalho em geral, os inquiridos atribuem um grau de importância muito semelhante a cada um deles (quadro 6), não se notando diferenças entre as apreciações dos licenciados em educação e as dos demais licenciados. A categoria status (trabalho que permite condição social privilegiada) continua a ser aquela a que todos os licenciados atribuem menor importância.

Os aspectos relacionados com trabalho seguro e estável, trabalho que permite oportunidades de progressão (carreira) e trabalho que permite rendimentos elevados (financeira) são mais valorizados quando se aprecia o trabalho em geral do que quando se considera a atividade profissional desempenhada pelos inquiridos.

Quadro 6 - Aspectos que mais valorizam no trabalho em geral

\begin{tabular}{|c|c|c|c|}
\hline Categorias & $\begin{array}{l}\text { Aspectos valorizados } \\
\text { Trabalho... }\end{array}$ & $\begin{array}{c}\text { Todos os licenciados } \\
\text { Média (desvio-padrão) }\end{array}$ & $\begin{array}{l}\text { Licenciados em Educação } \\
\text { Média (desvio-padrão) }\end{array}$ \\
\hline \multirow{2}{*}{ Carreira } & seguro e estável & $8,5(1,8)$ & $8,7(1,9)$ \\
\hline & que permite oportunidades de progressão & $8,4(1,6)$ & $8,4(1,8)$ \\
\hline \multirow{2}{*}{ Financeira } & que permite rendimentos elevados & $7,8(1,7)$ & $7,8(1,7)$ \\
\hline & que permite autonomia financeira & $9,2(1,3)$ & $9,2(1,5)$ \\
\hline Status & que permite condição social privilegiada & $5,8(2,1)$ & $6,1(1,9)$ \\
\hline \multirow{3}{*}{$\begin{array}{c}\text { Realização e /ou } \\
\text { desenvolvimento pessoal }\end{array}$} & que é interessante & $9(1,3)$ & $9(1,2)$ \\
\hline & que permite novos conhecimentos & $8,9(1,3)$ & $8,9(1,3)$ \\
\hline & que permite autonomia & $8,7(1,3)$ & $8,6(1,3)$ \\
\hline \multirow{2}{*}{ Altruísmo } & que permite ajudar os outros & $8,4(1,6)$ & $8,8(1,4)$ \\
\hline & que é útil à sociedade & $8,6(1,5)$ & $8,9(1,6)$ \\
\hline
\end{tabular}

Fonte: dados da pesquisa

Em síntese, a análise dos aspectos mais e menos valorizados no trabalho não revela grandes diferenças entre os licenciados em educação e os outros licenciados. Essa situação parece indiciar que a área de formação inicial não introduz variações significativas relativamente aos aspectos valorizados no trabalho. Apesar de ligeiras, as variações detectadas relacionam-se principalmente com a apreciação da atividade profissional desempenhada e menos com o trabalho em geral. As diferenças observadas dizem respeito à categoria altruísmo, mais valorizada pelos licenciados em educação e status, menos valorizado pelo mesmo grupo. Esse resultado é convergente com outros estudos que destacam as razões altruístas como sendo as mais comumente apontadas pelos professores para a escolha dessa profissão (SUMMERHILL; MYRNA, 1998; YONG, 1995).

\section{c) Caracterização da situação profissional}

No que diz respeito à situação profissional um ano e cinco anos após a conclusão da licenciatura (quadro 7), começamos por considerar a situação no mercado de trabalho. Note-se que, 
globalmente, com a passagem dos anos após o curso, aumenta a porcentagem de licenciados empregados e diminui a dos desempregados. Tal evolução é particularmente marcada no caso dos licenciados em educação, que registram uma porcentagem mais elevada de desempregados um ano após o curso, valor que se reduz bastante cinco anos após o curso.

Relativamente aos vínculos contratuais, observa-se que, um ano após o curso há mais licenciados em educação a exercerem atividade profissional sem contrato do que entre todos os licenciados. Esse valor reduz-se bastante quatro anos mais tarde, quando já poucos licenciados em educação estão sem contrato. No caso dos licenciados em geral, essa redução é menos acentuada.
Entre os que exercem atividade profissional com contrato de trabalho, a forma mais estável de contratação (contrato sem termo) é mais frequente entre os licenciados em geral do que entre os licenciados em educação nos dois momentos considerados. No entanto, em ambos os grupos, aumenta o número de contratos sem termo durante os cinco anos que se seguem à conclusão da licenciatura.

A respeito do rendimento mensal líquido, assinale-se que o mesmo tende a ser mais elevado cinco anos após a conclusão da licenciatura. Contudo, verifica-se que os licenciados em educação auferem rendimentos ligeiramente inferiores aos restantes licenciados, quer no primeiro ano de profissão, quer cinco anos após a conclusão da licenciatura.

Quadro 7 - Situação profissional um ano e cinco anos após o curso

\begin{tabular}{|c|c|c|c|c|c|}
\hline \multirow{2}{*}{\multicolumn{2}{|c|}{ Condição laboral }} & \multicolumn{2}{|c|}{ Todos os licenciados } & \multicolumn{2}{|c|}{ Licenciados em Educação } \\
\hline & & Após 1 ano & Após 5 anos & Após 1 ano & Após 5 anos \\
\hline \multirow{5}{*}{ Situação } & Empregado & $65,5 \%$ & $87,4 \%$ & $65 \%$ & $95 \%$ \\
\hline & Desempregado & $7,5 \%$ & $2,4 \%$ & $18 \%$ & $1 \%$ \\
\hline & Inativo & $7 \%$ & $3,7 \%$ & $5 \%$ & $2 \%$ \\
\hline & Estagiário & $18,9 \%$ & $1,1 \%$ & $12,5 \%$ & 0 \\
\hline & Bolseiro & $1,1 \%$ & $5,5 \%$ & 0 & $2 \%$ \\
\hline \multirow{3}{*}{ Tipo de contrato * } & Sem termo & $33,7 \%$ & $55,1 \%$ & $20 \%$ & $27,8 \%$ \\
\hline & Com termo & $55,6 \%$ & $38,3 \%$ & $53 \%$ & $65,6 \%$ \\
\hline & Prestação de serviços & $9,7 \%$ & $4,6 \%$ & $24,5 \%$ & $4,4 \%$ \\
\hline Sem contrato & & $22 \%$ & $15,5 \%$ & $30,8 \%$ & $5,3 \%$ \\
\hline $\begin{array}{l}\text { Rendimento líquido/mês } € \\
\text { (média) }\end{array}$ & & 957,4 & 1302,4 & 725,1 & 1062,5 \\
\hline
\end{tabular}

Fonte: dados da pesquisa

* Incluem-se apenas os tipos de contrato mais frequentes.

Em síntese, de um modo geral, os dados indiciam que a situação dos licenciados no mercado de trabalho é mais favorável com a passagem dos anos após a conclusão da licenciatura. No caso dos licenciados em educação, essa evolução global positiva parece ser ainda mais acentuada. Quando comparada com todos os licenciados, a situação desses é menos favorável um ano após o curso, mas encontram-se em uma situação mais vantajosa cinco anos após a licenciatura. Todavia, no que diz respeito à estabilidade dos vínculos 
contratuais e aos salários médios, constata-se que, mesmo cinco anos após o curso, os licenciados em educação estão em situação menos vantajosa quando comparados com os demais licenciados.

\section{Conclusão}

Os objetivos deste estudo consistiram em analisar o grau de satisfação na profissão, os aspectos mais e menos valorizados no trabalho e a situação profissional um ano e cinco anos após a conclusão da licenciatura, considerando os licenciados em educação em comparação com todos os licenciados das Universidades de Lisboa e Nova de Lisboa.

Os dados permitem constatar que, nas dimensões estudadas, não existem diferenças significativas entre os licenciados. Em uma primeira análise, essa constatação surpreende, tendo em conta que a profissão docente é caracterizada por especificidades relacionadas com a natureza da função de ensinar e sua respetiva complexidade, bem como com a miscigenação de elementos pessoais e profissionais no desempenho docente (ROLDÃO, 2007). Porém, os dados revelam que, do ponto de vista da situação profissional, da satisfação com a profissão e dos aspectos mais e menos valorizados no trabalho, os licenciados em educação partilham as mesmas características e opiniões dos licenciados das outras áreas de formação.

Assim, consideramos que os efeitos da socialização profissional associados à frequência do ensino superior são preponderantes relativamente às especificidades das diferentes áreas de formação disciplinar. Ou seja, os licenciados considerados no estudo parecem partilhar um conjunto de opiniões e apreciações sobre a profissão e o trabalho que são independentes da área de formação e da profissão que desempenham.

No que diz respeito a particularidades do grupo de licenciados em educação, destacamos, a partir dos dados analisados, a ligeira saliência que assumem os aspectos no trabalho que categorizamos como altruístas. Esse fato parece remeter, justamente, para a natureza da profissão docente, entendida como a função de ensinar e promover o desenvolvimento de outros. Também se verifica que esses licenciados se manifestam ligeiramente mais satisfeitos com a profissão. Eventualmente, tal consideração pode estar relacionada com a relação com os estudantes, que tem sido apontada como uma das razões de maior satisfação entre os professores (PERKING, 1991; CLARKE; KEATING, 1995).

Relativamente à situação profissional, não se verificam diferenças muito significativas entre os licenciados em educação e os outros licenciados. No entanto, constata-se uma tendência de precarização dos vínculos contratuais que é mais acentuada entre os licenciados em educação, os quais também tendem a auferir em média rendimentos ligeiramente inferiores. Em alguns estudos, a baixa remuneração é apontada como uma das fontes de insatisfação dos professores (PERKING, 1991; CLARKE; KEATING, 1995).

Além disso, a falta de apoio, o excesso de trabalho administrativo e o pouco acompanhamento do modo como se desenvolve o profissional nos primeiros anos da carreira são geralmente referidos como dificuldades sentidas pelos professores relativamente às suas condições de trabalho (PINTO et al., 2003; RUIVO et al., 2008; RHODES; NEVILL; ALLAN, 2004). Tendo em conta esse cenário e reconhecendo, com Flores e Day (2006), a importância do contexto na definição e redefınição da identidade dos professores nos primeiros anos de profissão, consideramos que seria importante aprofundar o modo como essas condições influenciam o desenvolvimento dos professores em fase de inserção profissional. Seria também pertinente explorar melhores formas de apoiar a aprendizagem profissional, bem como a construção da identidade profissional em início de carreira.

Como tem sido argumentado, tornar-se professor é um processo contínuo de desenvolvimento profissional, resultante da 
interação social significativa entre o professor em início de carreira e o seu ambiente profissional (ROTS; KELCHTERMANS; AELTERMAN, 2012; KELCHTERMANS, 2007). Seria importante compreender melhor como a biografia pessoal, a formação inicial e a cultura de escola se constituem como influências mediadoras chave na formação da identidade dos professores (FLORES; DAY, 2006).

A pesquisa indicou que é desejável a existência de um período de indução para professores na fase de inserção profissional após a licenciatura, como já acontece em vários países europeus (GONÇALVES; REIS; MESQUITA, 2011). No caso português, embora a implementação do período probatório (enquanto mecanismo de indução profissional para professores de qualquer nível de ensino) esteja prevista no estatuto da carreira docente, ela apenas foi concretizada no ano de 2009/10 (GONÇALVES; REIS; MESQUITA, 2011).

0 agravamento das condições de exercício profissional, bem como a natureza incipiente dos mecanismos de indução e acompanhamento da atividade profissional têm sido identificados como razões para muitos professores abandonarem a profissão (JOHNSON; BIRKELAND, 2003). De fato, diversas pesquisas revelam que os professores não têm intenção de permanecer toda a vida no ensino (AKKARI; TARDIF, 2011), em parte pelo fato de se verificar uma precarização crescente do emprego.

A partir do ano 2000, têm-se verificado a reforma de muitos professores e o aumento do número de alunos por professor, contribuindo para a diminuição dos empregos docentes (AKKARI; TARDIF, 2011). Essas tendências têm sido observadas em Portugal, em especial nos últimos dois anos, pelo que este trabalho tem de ser entendido como um ponto de partida que retrata a situação na primeira década do século XXI. Outras pesquisas são necessárias para aprofundar o conhecimento sobre os desafios que na atualidade se colocam à inserção profissional de professores de ensino básico e secundário.

\section{Referências}

AKKARI, Abdeljalil; TARDIF, Maurice. A inserção profissional no ensino: alguns pontos de referência sobre uma realidade complexa. In: GUIMARÃES, Célia Maria et al. (Org.). Formação e profissão docente. Araquara: Junqueira \& Marin, 2011. p. 124-141.

ALVES, Mariana Gaio. A inserção profissional de diplomados de ensino superior numa perspectiva educativa. Revista Europeia de Formação Profissional, n. 34, p. 31-44, 2005.

. A inserção profissional de graduados em Portugal: notas sobre um campo de investigação em construção. In: ALVES, Mariana Gaio; MARQUES, Ana Paula. (Eds.). Inserção profissional de graduados em Portugal: (re)configurações teóricas e empíricas. V. N. de Famalicão: Edições Húmus, 2010. p. 31-50.

BROOKHART, Susan. M.; FREEMAN, Donald J. Characteristics of entering teacher candidates. Review of Educational Research, n. 62, p. 37-60, 1992.

CHAVES, Miguel. Confrontos com o trabalho entre jovens advogados. As novas configurações da inserção profissional. Lisboa: ICS, 2010.

CLARKE, Robert; KEATING, W. Francis. A fresh look at teacher job satisfaction. ERIC Reproduction Service, n. ED, p. 391-795, 1995.

CORDEIRO-ALVES, Francisco. Estudo da satisfação/insatisfação dos professores efetivos do $3^{\circ}$ ciclo do ensino básico e do ensino secundário do distrito de Bragança. 1991. Dissertação (Mestrado em Ciências da Educação) - FPCE, Universidade de Lisboa, Lisboa, 1991.

DARLING-HAMMOND, Linda. Reshaping teaching policy, preparation, and practice: influences of the national board for professional teaching standards. Washington, DC: AACTE, 1999. 
DAY, Christopher; SACHS, Judyth. Professionalism, performativity and empowerment: discourses in the politics, policies and purposes of continuing professional development. In: DAY, Christopher; SACHS, Judyth. (Eds.), International handbook on the continuing professional development of teachers. Berkshire: Open University Press, 2004.

EREN, Altay; TEZEL, Kadir Vefa. Factors influencing teaching choice, professional plans about teaching, and future time perspective: a mediational analysis. Teaching and Teacher Education, v. 26, n. 7, p. 1416-1428, 2010.

FLORES, Maria Assunção; DAY, Christopher. Contexts which shape and reshape teachers identities. Teaching and Teacher Education, n. 22, p. 219-232, 2006.

FULLAN, Michael. The new meaning of educational change. 3. ed. New York: Teacher's College Press, 2001.

FURNHAM, Adrian. Personality at work. New York: Routledge, 1992.

; ERACLEOUS, Andreas; PREMUZIC, Tomas. Personality, motivation and job satisfaction: Hertzberg meets the big five. Journal of Managerial Psychology, n. 24, p. 765-779, 2009.

GONÇALVES, Teresa; REIS, Pedro; MESQUITA, Luciana. Contributos da primeira implementação do período probatório para 0 desenvolvimento profissional dos professores portugueses. In: GUIMARÃES, Célia Maria et al. (Coords.), Formação e profissão docente. Araraquara: Junqueira \& Marin, 2011. p. 167-186.

HARGREAVES, Andy. The changing nature of teachers' professionalism in a changing world. In: CHENG Yin Cheong; CHOW King Wai; TSUI, Kwok Tung (Eds.). New teacher education for the future: international perspectives. Tai Po, HK: Hong Kong Institute of Education. 2001.

HARGREAVES, Linda et al. The status of teachers and the teaching profession: views from inside and outside the profession: interim findings from the teacher status project. Research Report 755. London: DfES. 2006.

HARKER, Richard et al. Teacher 2000 project: an international study of teacher satisfaction, motivation and health. The New Zealand Report, 1998. Disponível em: <http://www.stats.govt.nz/Census/1996-census-data.aspx>. Acesso em: 2 jul. 2007.

HAYES, Sandra. Students' Reasons for entering the educational profession. ERIC document reproduction service n. ED366234, 1990. Disponivel em: <http://www.stats.govt.nz/Census/1996-census-data.aspx>. Acesso em: 5 ago. 2010.

HERZBERG, Frederick. A teoria motivação-higiene. In: MARQUES, Carlos Alves; PINA E CUNHA, Miguel (Coord.). Comportamento organizacional e gestão de empresas. Lisboa: Dom Quixote, 1996. p. 43-67.

HILDEBRANDT, Susan A.; EOM, Minhee. Foreign language teacher motivations for professionalization. The Journal of Language Teaching and Learning, n. 1, p. 39-53. 2011.

HUBERMAN, Michael. La vie des enseignants. Evolution et bilan d'une proféssion. Neuchâtel, Paris: Delacheaux \& Niestlé, 1989.

KUHLEN, R. G., \& JOHNSON, G. H. Change in goals with increasing adult age. Journal of Consulting Psychology, n. 16, p. 1-4, 1952.

JESUS, Saul Neves. Stress nos professores: sintomas, factores e estratégias de "coping". In: ALVES, José Matias (Org.). Professor, stress e indisciplina. Porto: Porto, 1998. p. 11-14.

Bem-estar docente. Perspectivas para superar o mal-estar dos professores. In: SILVA, Adelina Lopes; PINTO, Alexandra Marques. (Eds.). Stress e bem-estar. Lisboa: Climepsi, 2005. p. 167-184.

JOHNSON, Susan Moore; BIRKELAND, Sarah E. Pursuing a "sense of success": new teachers explain their career decisions. American Educational Research Journal, n. 40, p. 581-617, 2003.

et al. The experience of work-related stress across occupations. Journal of Managerial Psychology, n. 20, p. 178-187, 2005.

JÜTTE, Wolfgang; NICOLL, Katherine; OLESEN, Henning. S. Editorial: Professionalisation - the struggle within. European Journal for Research on the Education and Learning of Adults, v. 2, n. 1, p. 7-20, 2011.

KELCHTERMANS, Geert. Professional commitment beyond contract. Teachers' self understanding, vulnerability and reflection. In: BUTCHER, Jude; MCDONALD, Lorraine. (Eds.). Making a difference: challenges for teachers, teaching, and teacher education. Rotterdam: Sense, 2007. p. 35-53. 
LEITE, Carlinda. Percursos e tendências recentes da formação de professores em Portugal. Educação, v. 18, n. 57, p. 371-389, 2005.

LIU, Xiaofeng Steven; RAMSEY, Jase. Teachers' job satisfaction: analyses of the teacher follow-up survey in the United States for 2000-2001. Teaching and Teacher Education, n. 24, p. 1173-1184, 2008.

LOCKE, Edwin A. What is job satisfaction? Organizational Behavior and Human Performance, n. 4, p. 309-336, 1969.

MALDEREZ, Angi et al. Becoming a student teacher: core features of the experience. European Journal of Teacher Education, n. 30, p. 225-248, 2007.

MENON, Menon; PAPANASTASIOU, Elena; ZEMBYLAS, Michalinos. Examining the relationship of job satisfaction to teacher and organisational variables: evidence from Cyprus. International Studies in Educational Administration, v. 36, n. 3, p. 75-86, 2008.

MOE, Angelica; PAZZAGLIA, Francesca; RONCONI, Lucia. When being able is not enough. The combined value of positive affect and self-efficacy for job satisfaction in teaching. Teaching and Teacher Education, n. 26, p. 1145-1153, 2010.

OCDE. Creating effective teaching and learning environments: first results from TALIS. [S.I.: s.n.], 2009.

Building a high-quality teaching profession. Lessons from around the world. [S.I.: s.n.], 2011.

PEDRO, Neuza; PEIXOTO, Francisco. A satisfação profissional e auto-estima dos professores. Análise Psicológica, v. 24, n. 2, p. 247-262, 2006.

. Auto-eficácia e satisfação professional dos professores: colocando os construtos em relação num grupo de professores do ensino básico e secundário. Revista de Educação, v. 18, n. 1, p. 23-47, 2011.

PERKINS, Cline M. A study to investigate experienced teachers job satisfaction and the teachers' perception of their principals' leadership style. Doctoral Dissertation, The University of North Carolina at Chapel Hill. Dissertation Abstracts International, v. 52, n. A4171, 1991.

PINTO, Alexandra Marques et al. Stress profissional em professores portugueses: incidência, preditores e reacção ao burnout. Psychologica, n. 33, p. 181-194, 2003.

PRATTE, Richard; RURY, John L. Teachers, professionalism, and craft. Teachers College Record, v. 93, n. 1, p. 59-72, 1991.

REEVE, Johnmarshall; BOLT, Elisabeth; CAI, Yi. Autonomy-supportive teachers: how they teach and motivate students. Journal of Educational Psychology, v. 91, n. 3, p. 537-548, 1999.

RHODES, Chistopher; NEVILL, Allan; ALLAN, Jo. Valuing and supporting teachers: a survey of teacher satisfaction, dissatisfaction, morale and retention within an English local education authority. Research in Education, n. 71, p. 67- 80, 2004.

RICHARDSON, Paul; WATT, Helen. 'I've decided to become a teacher': influences on career change. Teaching and Teacher Education, n. 21, p. 475-489, 2005.

RINKE, Carol R. Understanding teachers' careers: linking professional life to professional path. Educational Research Review, n. 3, p. 1-13, 2008.

ROLDÃO, Maria do Céu. Função docente: natureza e construção do conhecimento profissional. Revista Brasileira de Educação, v. 12, n. 34, p. 94-103, 2007.

ROTS, Isabel; KELCHTERMANS, Geert; AELTERMAN, Antonia. Learning (not) to become a teacher: a qualitative analysis of the job entrance issue. Teaching and Teacher Education, v. 28, n. 1, p. 1-10, 2012.

RUIV0, João et al. Ser professor: satisfação profissional e papel das organizações de docentes: um estudo nacional. Castelo Branco: Instituto Politécnico de Castelo Branco; ANP, 2008.

SKAALVIK, Elinar M.; SKAALVIK, Sidsel. Teacher job satisfaction and motivation to leave the teaching profession:relations with school context, feeling of belonging, and emotional exhaustion. Teaching and Teacher Education, n.27, p. 1029-1038,2011. 
SPECTOR, Paul.E. Job satisfaction: application, assessment, cause and consequences. Thousand Oaks: Sage, 1997.

SPITTLE, Michael; JACKSON, Kevin; CASEY, Meghan. Applying self-determination theory to understand the motivation for becoming a physical education teacher. Teaching and Teacher Education, v. 25, n. 1, p. 190-197, 2009.

STIEGELBAUER, Suzanne. Why we want to be yeachers: new teachers talk about their reasons for entering the profession. In: ANNUAL MEETING OF THE AERA, 1992, SF, CA.

SUMMERHILL, Arlene et al. High school seniors' perceptions of a teaching career. Journal of Teacher Education, v. 9, n. 3, 1998.

WANG, Hsiou-Huai. Why teach science? Graduate science students' perceived motivations for choosing teaching as a career in Taiwan. International Journal of Science Education, v. 26, n. 1, p. 113-128, 2004.

WATT, Helen. M. G.; RICHARDSON, Paul.W. Motivations, perceptions, and aspirations concerning teaching as a career for different types of beginning teachers. Learning and Instruction, v. 18, n. 5, p. 408-428, 2008.

WEISS, Howard. M. Deconstructing job satisfaction: separating evaluations, beliefs and affective experiences. Human Resource Management Review, n. 12, p. 173-194, 2002.

YONG, Bob Chui Seng. Teacher trainees' motives for entering into a teaching career in Brunei, Darussalam. Teaching and Teacher Education, v. 2, n. 3, 1995.

ZABEL, Robert. H.; ZABEL, Mary Kay. Revisiting burnout among special education teachers: do age, experience, and preparation still matter? Teacher Education and Special Education, v. 24, n. 2, p. 128-139, 2001.

ZUMWALT, Karen; CRAIG, Elisabeth. Who is teaching? Does it matter? In: COCHRAN-SMITH, Marilyn et al. (Eds.). Handbook of research on teacher education: enduring questions in changing contexts. 3. ed. New York: Routledge/Taylor \& Francis Group \& ATE, 2008, p. 134-156.

Recebido em: 05.12.2012

Aprovado em: 26.03.2013

Mariana Gaio Alves é licenciada em sociologia, mestre e doutora em Ciências de Educação pela Faculdade de Ciências e Tecnologia da Universidade Nova de Lisboa (FCT/UNL). Atualmente é professora auxiliar na FCT/UNL e investigadora na UIED (Unidade de Investigação Educação e Desenvolvimento) na mesma faculdade.

Nair Rios Azevedo é doutora em Ciências da Educação pela Faculdade de Ciências e Tecnologia da Universidade Nova de Lisboa. Tem graduação em psicologia pelo Instituto Superior de Psicologia Aplicada (Lisboa), pós-graduação em Educação pela Universidade de Stanford (EUA) e Mestrado em Desenvolvimento da Criança pela FMH da Universidade Técnica de Lisboa. É investigadora auxiliar na Unidade de Investigação Educação e Desenvolvimento (UIED), da Faculdade de Ciências e Tecnologia, Universidade Nova de Lisboa.

Teresa N. R. Gonçalves é doutora em Ciências da Educação pela Universidade de Sevilha. Tem graduação em filosofia pela Universidade de Lisboa, mestrado em Filosofia pela Universidade Católica de Lovaina e diploma de Estudios Avanzados pela Universidade de Sevilha. É investigadora auxiliar na Unidade de Investigação Educação e Desenvolvimento (UIED), da Faculdade de Ciências e Tecnologia, Universidade Nova de Lisboa. 